\title{
LA CONDICIÓN JUVENIL CONTEMPORÁNEA EN LA CONSTITUCIÓN IDENTITARIA
}

DINA KRAUSKOPF ${ }^{*}$

\section{RESUMEN}

El tiempo de ser joven identitariamente varía entre estratos, culturas y clases sociales, debilitándose el concepto de moratoria y evidenciándose la existencia de la premura psicosocial. La moderna prolongación de la vida y las sociedades de riesgo, se acompañan de incertidumbre, valoración de la individualización, formas flexibles de estabilidad y búsqueda de inserciones sociales con potencial innovativo. En la estructuración identitaria se diferencia entre individuación, individualización, identidad e individualismo. Se distingue la identidad multicéntrica, sus relaciones con las identidades sociales y la presencia de identidades parciales. En la participación juvenil se puntualiza la redistribución del poder, las nuevas relaciones de género e intergeneracionales.

PALABRAS CLAVE: JUVENTUD, IDENTIDAD, MODERNIDAD

* Profesora Emérita de la Universidad de Costa Rica. Investigadora y Consultora Internacional en Políticas de Juventud.

Correo electrónico: dina.krauskopf@gmail.com. 


\title{
A CONDIÇÃO JUVENIL CONTEMPORÂNEA NA CONSTITUIÇÃO IDENTITÁRIA
}

\begin{abstract}
RESUMO
O tempo de ser jovem, do ponto de vista identitário, varia conforme estratos, culturas e classes sociais, enfraquecendo o conceito de moratória e evidenciando a existência da urgência psicossocial. A moderna extensão da vida e as sociedades de risco são acompanhadas por incerteza, valorização da individualização, formas flexíveis de estabilidade e busca de inserções sociais com potencial inovador. A estruturação identitária diferencia-se entre individuação, individualização, identidade e individualismo. Distingue-se a identidade multicêntrica, suas relações com as identidades sociais e a presença de identidades parciais. $\mathrm{Na}$ participação juvenil, pontua-se a redistribuição do poder, as novas relações de gênero e intergeracionais.
\end{abstract}

PALAVRAS CHAVE: JUVENTUDE, IDENTIDADE, MODERNIDADE

\section{THE CONTEMPORANEAN YOUTH CONDITION AND THE CONSTITUTION IDENTITY}

\begin{abstract}
Youth identity varies between social levels, cultures and classes, being debilitated by the moratorium concept and demonstrating the existence of psychosocial pressure. The modern prolongation of the life and the societies of risk, are accompanied by uncertainty, valuation of individualisation, flexible forms of stability, and search of social insertions with innovative potential. In the identity structuring difference between individualisation, identity and individualism. One distinguishes the multi-centre identity, its relations with the social identities and the presence of partial identities. In youth participation the redistribution of the power, and the new intergenerational relations of generics is emphasized
\end{abstract}

KEY WORDS: YOUTH, IDENTITY, MODERNITY 


\section{INTRODUCCIÓN}

LA GLOBALIZACIÓN Y LA modernización han prolongado la vida en el contexto de cambios acelerados, con la consecuente modificación y ruptura en las secuencias del recorrido existencial. La moderna prolongación de la vida repercute en que la fase juvenil sea un período cada vez más extenso, se adquiera sentido e identidad por sí mismo y se desarrollen nuevas subjetividades. Las identidades de los jóvenes se han fortalecido y no se consideran meros seres en transición. El dominio directo de la familia y el sistema escolar sobre el entorno han disminuido. Han pasado, en mayor o menor grado, a ser redes más abiertas, atravesadas por otras agencias socializadoras y por las influencias multiculturales.

La velocidad de los cambios incide en la rápida obsolescencia de conocimientos y técnicas, aparecen expresiones culturales y ámbitos de conocimiento manejados por las nuevas generaciones, las distancias generacionales de los jóvenes con los adultos se modifican y resignifican. Cambia la potencialidad de un proyecto fijo y predeterminado, y se da mayor sentido a inserciones sociales con potencial innovativo.

El desarrollo juvenil existe en estructuras de oportunidades y condiciones económico-políticas predominantemente duales, que se traducen en la heterogeneidad de las juventudes. La homogeneización de la percepción oficial de la juventud se caracteriza por la debilidad en el enfoque de género, cultura, etnia, residencia rural/urbana y estrato económico. La alfabetización tradicional claramente no es un insumo suficiente para el desarrollo; existe el alfabetismo cibernético, de cuyos avances y ventajas van quedando alejados los grupos excluidos. Señala Lechner (1998) que ello contribuye a la dualidad social: mayor aproximación y articulación internacional para los grupos de más recursos económicos y mayor aislamiento para los sectores pobres.

El tiempo de ser joven identitariamente varía entre estratos, culturas y clases sociales. La sociedad está más abierta a la movilidad individual, mientras se reducen los canales para la movilidad estructural. Para el desarrollo de los jóvenes que crecen en exclusión, la prolongación de la vida no es una promesa existencial. Salazar ha identificado la cultura de muerte, donde se acortan las distancias entre vivir y morir, como es el caso de los jóvenes sicarios y los miembros de las ma- 
ras. ${ }^{1}$ Para este autor, la violencia muda de los jóvenes es la imposibilidad de articular algún discurso sobre las rupturas que viven.

Como señala Beck (1998), las sociedades contemporáneas se han constituido en sociedades de riesgo. Ello se debe a que los peligros producidos socialmente sobrepasan la seguridad. La brecha social lleva a que la riqueza se acumule en los estratos más altos, mientras que los riesgos se acumulan en los más bajos y en todos los niveles es necesario que los individuos se apoyen más en sus capacidades personales y dependan menos de las instituciones.

La nueva condición juvenil destaca por la valoración de la individualización, la avidez por multiplicar experiencias vitales, el retraso en la autonomía económica, un ejercicio más temprano de la sexualidad y menor urgencia por adquirir la condición adulta. La interconectividad amplía la espacialidad, se requiere menos presencia física para establecer intercambios, lo que impacta las relaciones sociales, materiales y simbólicas, y el usuario puede relacionarse con múltiples actores en distintos contextos. La informática produce realidades virtuales que abren nuevas rutas para elaboración de la identidad y las cosmovisiones.

De este modo, se demandan nuevas estrategias de enfrentamiento de las situaciones. Una consecuencia es que cada individuo tiene que aportar más esfuerzos propios que antes, para poder moverse en el sistema de coordenadas del mundo que le rodea y para construirse una identidad mínimamente estable en mundos de vida individualizados. La individualización creciente empezó con la modernidad, pasando de un modelo cultural industrial a un modelo cultural identitario, fundado en los principios de independencia y autorrealización individuales (Beck-Gernsheim, 1990).

La actualización de identidades sociales y de los proyectos de los jóvenes se fortalece con la expresión cultural como campo político y los logros de la participación efectiva, apareciendo nuevas expresiones de lo colectivo que integran condiciones de la individualización con formas propias de empoderamiento social. Serna (1998) destaca que las acciones juveniles procuran, actualmente, cambiar el presente con autonomía e identidad para derivar de allí el cambio social. Se prioriza el sentido de vida, la acción inmediata, la búsqueda de la efectividad palpable de su acción. El respeto a la diversidad y a las individualidades se constituye en el centro de las prácticas y ello implica que los

1 Término que surge en Centroamérica, originariamente en Guatemala, para designar las pandillas juveniles. 
jóvenes prefieren voceros a representantes y no se sienten afines a estructuras sociales burocráticas. Cabe destacar, por otro lado, la globalización del enfoque de derechos, que contribuye a las transformaciones que se han dado en los contenidos de la participación juvenil y ciudadana.

\section{FORMAS CONTEMPORÁNEAS DE ORGANIZACIÓN IDENTITARIA}

La actual preocupación del individuo por sí mismo no debe confundirse, como ocurre frecuentemente, con el individualismo en el sentido de egoísmo. Para un número creciente de personas, especialmente entre los jóvenes, «tener éxito en la vida» es llegar a ser uno mismo, autorrealizarse, seguir su propio camino. Es necesario, entonces, descubrir para lo que está hecho uno: su pasión, su vocación, su fuego sagrado. El «sé tú mismo» está convirtiéndose en una orden cultural y social que provoca el mayor autocuidado, «sentirse bien en su cabeza, en su corazón, en su cuerpo» (Bajoit, 2003:118). Ello significa que la plenitud personal y la de los otros se está convirtiendo en un valor principal e incide en las conductas relacionales de la vida social. En este contexto la permisividad tiene que ver con la relación consigo mismo y la tolerancia, con la relación con los otros.

Beck se pregunta si los individuos, con todo su deseo de autodeterminación, no son sino agentes de un cambio profundo. ¿Se trata de indicios, de augurios de una nueva era, de una nueva relación entre individuo y sociedad aún por encontrar e inventar? ¿De una nueva forma de sociabilidad ya no deducible de modelos y recetas antiguas? ¿Será que perspectivas procedentes de lo individual y lo biográfico se unen y se mantienen a través de nuevos acuerdos, preguntas y razonamientos que se consiguen cuestionando la fuerza centrífuga de la biografía? (Beck-Gernsheim, 1990). La individualización significaría, por lo tanto, que la biografía del ser humano se despega de los modelos y de las seguridades tradicionales, de los controles ajenos y de las leyes morales generales $\mathrm{y}$, de manera abierta y como tarea, se adjudica a la acción y a la decisión de cada individuo. La individualización significa que los seres humanos se liberan de los roles de género internalizados, como estaban previstos en el proyecto de construcción de la sociedad industrial, para la familia nuclear. Al mismo tiempo se agudiza la suposición que están obligados a construirse, bajo pena de perjuicios materiales, una existencia propia a través del mercado labo- 
ral, de la formación y de la movilidad y, si fuera necesario, en detrimento de las relaciones familiares, amorosas y vecinales (op. cit.: 2021). Landa y Cruz (2006) observan que el trabajo individualiza y puede desvincular de situaciones e intereses compartibles. Por otro lado, la individualización se presenta como un requerimiento para la integración social (INJUV, 2004). En diferentes entornos, el proceso de individualización avanza a velocidades distintas y no necesariamente en la misma dirección.

Por lo tanto, la individualización no es lo mismo que el individualismo, la individuación ni la identidad. Debemos diferenciar y definir estos términos que, a menudo, se hacen equivalentes y tienen significados claramente distintos. El individualismo, al confundirse frecuentemente con la individualización, contribuye a la estigmatización de los jóvenes contemporáneos. El individualismo es la organización conductual del individuo que da prioridad a sus intereses con desinterés y a menudo desconsideración hacia los demás.

Las identificaciones son un medio primario de la especie humana para transmitir y aprehender patrones y valores culturales, y brinda fuentes de organización de diferentes posibilidades de conducta del individuo. Se han iniciado en la niñez y pueden ocurrir sucesivamente, incorporando ciertas características a través de la vinculación afectiva y la socialización. Se elaboran y sintetizan en contextos de experiencia que brindan las condiciones dominantes, las particularidades de los sujetos, la índole de conflictos y gratificaciones con grupos del escenario social en que se desenvuelven (Krauskopf, 1994, 1996).

Mediante los procesos de individuación, la construcción de la identidad toma insumos de las identificaciones previas y va más allá de éstas para entrar en procesos de individuación con diferenciación de las figuras significativas y lograr la identidad personal conocida en ciertos ámbitos como identidad del yo. Se experimenta como una vivencia básica de conexión interna que articula el sentido de vida, el reconocimiento recibido, las pertenencias sociales del sujeto. Toda identidad involucra reproducción social y proyección social. La identidad da sustento a la expresión de comportamientos, sentimientos y relaciones dentro de contextos sociales específicos.

Todo individuo tiene que realizar durante la fase juvenil su propia y original síntesis de los modelos, identificaciones e ideales, a menudo incompatibles o contradictorios, que nuestras sociedades ofrecen. Es necesario un sentimiento positivo de identidad, para lo cual los jóvenes demandan el reconocimiento social y luchan por su reputación. 
Como señala Chon (2003), la identidad integra cómo me asumo y cómo me asumen. Consideramos que estas características se dan en el marco de la individualización, pues los jóvenes requieren ser gestores y cogestores de su desarrollo e integración social. Bajoit (2004) destaca lo que denomina los mandamientos de la individualización: que sean ellos mismos, que se realicen, que sean lúcidos y sinceros con sí mismos, que elijan lo que quieren ser, no importa equivocarse, hay que descubrir y realizar los sueños, alcanzar el máximo de placer, no está bien sufrir, no hay que dejar de velar por su seguridad, ser libre, pero sin errar pues les espera la muerte social, ser un supernumerario.

\section{IDENTIDAD PERSONAL E IDENTIDAD SOCIAL: NUEVAS FORMAS DE ARTICULACIÓN}

Hasta ahora han predominado los análisis compartimentados sobre las manifestaciones individuales y sociales de las identidades juveniles. La interrelación entre ambas ha sido escasamente tratada en el estudio de la identidad, posiblemente porque los primeros provienen de la psicología y los segundos de los estudios culturalistas y sociológicos. Ya no es posible comprender las identidades de modo unívoco y lineal a partir de sus manifestaciones.

Poitevin (2003) enfatiza que la juventud es una categoría en construcción, lo cultural se expresa en pertenencias complejas, heterogéneas, segmentadas y las identidades conducen a relaciones múltiples que han sido frecuentemente cosificadas alrededor de las prácticas. Reguillo (1999) desarrolla la metáfora del hipertexto, que permite acercarse y comprender los procesos de configuración simbólica y social de las culturas juveniles. Señala que estas características juveniles han ocasionado dificultades tanto para los estudiosos de las culturas juveniles como para las instituciones de control social, al partir de un enfoque que fija los sujetos para comprender el sentido de sus prácticas.

Para Bajoit (2003) lo que está cambiando es la relación del individuo con lo social; los hechos sociales no pueden ser analizados como cosas, porque nunca se reducen a ellas, solo existen expresadas en conductas sociales de los individuos y grupos. Por otro lado, agregamos, estas conductas nos llevan a inferir las identidades a partir de la configuración de las constantes externas. No hay observación directa de las identidades.

Las identidades juveniles se elaboran en la vinculación de procesos subjetivos e intersubjetivos con los cuales los jóvenes dan forma 
concreta a su condición de sujetos sociales y actores históricos. Las identidades sociales se manifiestan en la relación entre el individuo y la colectividad. Xón (2003), analizando las identidades juveniles indígenas, precisa que ello incluye lo político, lo cultural y lo social. Lungo (2003) observa en las juventudes salvadoreñas de izquierda el carácter multidimensional que se manifiesta en los intereses y en la acción colectiva de los jóvenes organizados, lo que atribuye a las transformaciones sociales desarrolladas en los últimos años, sobre todo con el auge de la globalización económica, tecnológica y cultural.

Consideramos que las identidades en la modernidad propenden a una organización multicéntrica que demanda un abordaje integral e innovador en el reconocimiento de las prácticas juveniles. Solo así se podrá abandonar la frecuente afirmación de que las identidades juveniles son situacionales, múltiples y transitorias (decir que son múltiples o esquizofrénicas, involucra desintegración personal). La identidad, al ser multicéntrica, permite tener una vivencia de conexión aún en la transformación así como en sus diversas expresiones en distintos contextos y demandas interpersonales y sociales.

Gómez (2003) advierte que las investigaciones sobre la identidad juvenil gravitan alrededor de una identidad determinante, señalando que es importante tener claro que las identidades sociales forman parte de un funcionamiento más global. Los jóvenes han sido pensados como estudiantes, como guerrilleros o subversivos, como drogadictos, como mareros o delincuentes. Sin embargo, al rescatar el valor de la pluralidad de las membresías, se amplía el espectro de la construcción de la identidad y se relativiza la tendencia a generalizar alguna identidad social, como negativa o positiva. Martín Barbero afirma que, en la actualidad, se produce una configuración de identidades juveniles marcada menos por la continuidad que por la amalgama (1998:30).

Landa y Cruz (2006) destacan que la dinámica de los contextos espacio/temporales en los que se desenvuelven las identidades juveniles, con la integración a formas contemporáneas de congregaciones colectivas, les lleva a resignificar y reemplazar símbolos y acciones, o permitir su coexistencia sin causarles conflictos. Así, «las identidades flexibles bien pueden ser un puerto de salida para reconocer un continente de lo juvenil más amplio de lo que se había pensado» (Landa y Cruz, 2006:199). A modo de ejemplo, pensemos en las simplificaciones que se hacen cuando se analizan jóvenes que están insertos en ciertas grupalidades juveniles que son llamadas tribus urbanas, dejando otros aspectos de sus identidades y trayectorias reducidos con este abordaje. 
La identidad social podemos entenderla como el producto de la interacción de distintos componentes identitarios que se articulan para insertarse en determinado espacio sociopersonal y dan sustento a la expresión de comportamientos y relaciones. Cobran sentido dentro de contextos específicos, reflejando la tensión entre el desarrollo idiosincrásico y la dinámica de la situación (Krauskopf, 2007). Cuando uno de los componentes identitarios pasa a ser el referente totalizador del individuo se produce una organización parcial rígida y continua que no integra flexiblemente otros referentes, caso en el que consideramos apropiado hablar de identidades parciales. Un ejemplo lo encontramos en los portadores de posiciones fundamentalistas.

\section{LA INCERTIDUMBRE COMO CONTEXTO EN LA CONSTRUCCIÓN DE IDENTIDADES}

La aceleración de los cambios demanda integrar, tanto en el plano individual como colectivamente, la parte de lo que es imprevisible o indeducible como fuente de acontecimiento decisivos (Maffesoli, 2001:10). Las estrategias derivadas del paradigma del futuro esperable (supuestamente encarnado en la adultez actual), como foco orientador de la preparación y educación de la juventud, ven reducida su efectividad ante la falta de predictibilidad que se desprende de la interacción rápida de factores a escala mundial. La imprevisibilidad contribuye a la priorización del presente. Existe dificultad para percibir un horizonte estructurado que estimule la depositación de la vida en el futuro adulto y la preparación para éste sin participar del presente. Las personas en la fase juvenil ya no corresponden a un preproyecto de futuro y requieren enfrentar los cambios y la incertidumbre con imaginación y alta capacidad de aprendizaje (Krauskopf, 2003). Ello afecta el campo educacional donde será necesario también dar énfasis a aprender a aprender, aprender a ser, aprender a hacer y competencias para gestionar el avance de los conocimientos e intereses.

El ámbito económico contribuye con la acumulación flexible, la aceleración del tiempo de rotación en el consumo, lo que acentúa la fugacidad de los productos, la transformación acelerada de su estética y utilidad (Landa y Cruz, 2006). El manejo de la presencia de lo efímero e incierto es parte de las gestiones de la individualización para enfrentar la inestabilidad, pues vivir en un crisol de contradicciones, cambios y diversidad de insumos no puede ser provisional durante mucho tiempo. Se hace necesario desarrollar estrategias de articulación interna y de 
relación con el mundo externo para incorporar la paradoja social que Reguillo ha llamado «la incertidumbre como única certidumbre».

Esto nos lleva a afinar los conceptos sobre la constitución de la estabilidad como eje de la identidad contemporánea, puesto que se hace necesario basarla en la flexibilidad, la incorporación de la multicentralidad, la valoración de la diversidad de alternativas, recursos e intereses para nutrir el sentido del yo. Serán el aprendizaje permanente, los conocimientos, destrezas emocionales y sociales de amplia aplicabilidad, los que permitan enfrentar los cambios y las vicisitudes con imaginación, participación y expresión transformadora.

La modernización y la globalización van acompañadas de la secularización, pluralización de los mundos de la vida, competencia de valores y sistemas religiosos. Así se han disuelto muchos referentes que daban al individuo una visión del mundo, un contexto productor de sentido, un arraigo de la propia existencia dentro de un cosmos más global. Ello puede dar paso a una incertidumbre moral que tiene consecuencias psicológicas, sobre todo si se suma a otras formas de incertidumbre material — problemas de empleo, de exclusión social y de ingresos, riesgo de enfermedades, amenazas sobre el medio ambiente-, institucional —el sentido de que el mundo político no es confiable-, social — reducción de la solidaridad, fragilidad de los lazos sociales, soledad-(Beck-Gernsheim, 1990:73).

En este mundo que los insta a ser ellos mismos, pero no les ofrece los medios de realización personal, son muchos los que pierden la brújula. No pocos se inventan vocaciones identitarias poco realistas y fracasan. Otros no saben lo que quieren y están convencidos de que «no vale la pena intentar». Se necesitan todos los recursos de la flexibilidad, la aceptación de sí mismo y un sentido de vida positivo para manejar los fracasos resultantes. También se producen perturbaciones psicológicas que generan en muchos un sentimiento de vacío, de aburrimiento, de anomia. Dichas perturbaciones conciernen a menudo a la identidad, las relaciones sociales y la forma de actuar: «bastantes individuos, desorientados por la velocidad y la profundidad de los cambios, ya no saben muy bien ni lo que son, ni lo que quieren ni cómo conducir sus relaciones con los otros» (Bajoit, 2003:120). Parte del repertorio para olvidar la pregunta del sentido, es el consumo y para responder a la ausencia de un repertorio conductual satisfactorio, se encuentra la violencia.

También el tiempo presente se enaltece, con una pérdida de profundidad y una exaltación de los impactos instantáneos, las experien- 
cias intensas, los estilos cambiantes, las aventuras riesgosas, innovadoras, fugaces, el logro de apariencias que brinden visibilidad en la invisibilidad citadina. Un ejemplo son las prácticas culturales de los grafiteros, que deben actuar en poco tiempo, la acción misma es muy importante y su arte es efímero en su duración. No tienen garantía de permanencia, pero logran dejar huella de su presencia y ganan protagonismo (Landa y Cruz, 2006). Es el instante eterno de que habla Maffesoli.

\section{LAS RELACIONES GENERACIONALES: ENCUENTROS Y DESENCUENTROS EN LA ELABORACIÓN IDENTITARIA}

Las condiciones existenciales actuales llevan a enfrentar muchas dificultades en distintos momentos del ciclo vital, y no es raro encontrar personas de cincuenta años atravesando una fase de moratoria que antes sólo se atribuía a la adolescencia. El signo juventud se valoriza cada vez más en los adultos. Así como en tiempos pretéritos los jóvenes próceres procuraban verse respetablemente mayores, y por lo tanto experimentados y sabios, ahora se produce el fenómeno de la juvenilización, donde los mayores procuran verse jóvenes, por consiguiente flexibles, innovadores, con opciones abiertas ante la prolongación de la vida y dispuestos a no dejarse desplazar por los jóvenes. La trama del desarrollo identitario se teje en el contexto de nuevas relaciones de género e intergeneracionales así como con las dificultades que generan el fraccionamiento social, la exclusión y la pobreza. Las distancias generacionales con los adultos se resignifican y modifican. Ahora los jóvenes saben cosas que los adultos no saben. ${ }^{2} \mathrm{El}$ ritmo de difusión de los conocimientos rompe las antinomias (el que sabe versus el que se prepara) que dejaban como fase de relevo a los jóvenes de los grupos incluidos.

En nuestras sociedades se ha producido una fuerte tensión antagonista entre la adultez y la juventud. Por un lado está la resistencia de los adultos a aceptar el protagonismo y la toma de decisiones por parte de los jóvenes; y por otro lado, la resistencia juvenil a través de la desconexión activa que expresa la desconfianza frente a una institu-

2 La falta de reconocimiento de este nuevo balance, muchas veces se expresa en los bloqueos generacionales (término aportado por el sociólogo uruguayo Julio Bango), una especie de discurso paralelo entre jóvenes y adultos que afecta el sistema educativo y la vida familiar e incluso el ámbito público. 
cionalidad donde es notoria la debilidad de propuestas realmente incluyentes para las juventudes.

La idea de la moratoria psicosocial se instaló a partir de la postergación de la acción y la toma de decisiones como la estrategia necesaria en el período de preparación juvenil para la adultez. Las acciones que implementan las propuestas de intervención y atención para analizar el período juvenil requieren despojarse de estos esquemas que provienen de épocas en que la tradición era la respuesta para el futuro. La ineficacia de dichos instrumentos rigidiza y agrava las respuestas de los mayores, devalúa las capacidades juveniles, incrementa la discriminación etaria y las distancias generacionales entre jóvenes y adultos. En la actualidad, las dos generaciones están preparándose permanentemente, pueden trabajar de manera colaborativa y requieren considerar la flexibilidad frente a los cambios por sobre la irreversibilidad de los pasos dados.

\section{Posicionamiento SOCIAL DE LAS JUVENTUDES Y CIUDADANÍA}

La gran mayoría de los menores de edad de la región latinoamericana, que viven en exclusión, están invisibilizados y enfrentan la premura psicosocial $^{3}$ (opuesta a la moratoria) en el cumplimiento de responsabilidades supuestamente adultas y con ausencia de oportunidades. Esta premura se intensifica a partir de la pubertad, momento del ciclo vital que parece legitimar su responsabilidad de procurar la subsistencia y aportar a sus familias. Liebel (1992) concluye que, en sociedades jóvenes donde la esperanza de vida es menor, las personas cumplen períodos más breves de actividad y son relevadas más rápidamente por las generaciones siguientes.

Por otro lado, en el imaginario social, a los jóvenes aún se los considera los adultos del mañana, para lo cual deben alcanzar la meta: una adultez concebida con un futuro ya estructurado. Ello privilegia la preparación, o subordinación por sobre el aporte participativo del sujeto juvenil ciudadano, y da lugar a representaciones sociales desvinculadas de la experiencia y de la cosmovisión juvenil. En cierto sentido marginan a los jóvenes, invisibilizan sus aportes e incrementan la conflictividad intergeneracional.

Un agravante es que el concepto de transición se utiliza como equivalente de transitoriedad. Implícitamente esto niega el reconoci-

3 Premura psicosocial: término acuñado por De La Garza y otros (1977). 
miento de los jóvenes como sujetos sociales del presente y se destaca su incompletud (inmadurez, inexperiencia). Son invisibilizados en cuanto a sus aportes y visibilizados cuando perturban el orden social. Ello da paso fácilmente a la visión de la juventud problema que se apoya en un énfasis estigmatizante y reduccionista de la juventud (Krauskopf, 2003). Martín-Barbero destaca que durante siglos, ser joven se identificó con la negación de la responsabilidad y la productividad, así como lo popular se constituyó por la exclusión de la riqueza, la educación y la cultura. Lutte observa que en las sociedades en que existen jerarquías claramente definidas, especialización del trabajo y diferencias socioeconómicas, la adolescencia es «una fase de marginación que deriva de las estructuras sociales fundadas en la desigualdad» (1991:35).

Como señalan Landa y Cruz (2006:183), lo económico no está desligado de la dimensión cultural, sino que guarda una dimensión espacio/tiempo y causa/efecto variables. Los jóvenes en exclusión económica y social deben vivir al margen de los referentes socioinstitucionales que dan identidad y pertenencia integradora. Las características de sus expresiones identitarias reflejan las circunstancias de la exclusión y la desautorización moral que los rodea, su anonimato como actores sociales.

Un cambio histórico se ha producido con la reciente inclusión democrática de niños y adolescentes, al ampliar los atributos de la ciudadanía con la promoción de los derechos humanos. Sin embargo, la aceptación de los derechos de los jóvenes sufre toda suerte de embates. La cultura poco participativa provocada por los conflictos armados y la violencia ha sometido a los adolescentes y jóvenes a situaciones difíciles y la discriminación por edad afecta a los sectores jóvenes.

Con la Convención Internacional de los Derechos del Niño, que entiende la ciudadanía «como la adquisición de derechos que permiten a las personas menores de edad pronunciarse e involucrarse en las decisiones que afectan sus vidas», la ciudadanía ha dejado de ser entendida como el derecho al voto adquirido con la mayoría de edad y ya no se constituye solo por la posibilidad formal de ejercerlo. Queda un largo camino para el reconocimiento efectivo de las personas jóvenes como sujetos de derecho y para el ejercicio de las facetas ciudadanas que ese reconocimiento implica: conocer, construir, participar. Por ello, ha surgido la Convención Iberoamericana de los Derechos de los Jóvenes, ratificada ya por siete países, por lo que se constituye en un Tratado Internacional y en un nuevo marco para el desarrollo de las 
políticas juveniles. De este modo, se pueden enriquecer las relaciones entre el Estado y los actores sociales juveniles.

\section{CONSIDERACIONES FINALES}

Hemos realizado un recorrido sobre las nuevas condiciones de la vida contemporánea, las importantes transformaciones de la modernidad y la globalización, los requerimientos que emergen y las constituciones identitarias. Las juventudes no solo enfrentan los cambios sino, también, la dificultad de sus entornos para reconocer sus actuales características, capacidades, derechos, desafíos y necesidades. La construcción de lo juvenil se da en contextos con mayor o menor grado de multiculturalidad y multilocación, estructuras de oportunidades enraizadas en condiciones económico-políticas con una distribución predominantemente dual. El mundo cibernético es parte de un cambio cultural que agudiza las diferencias y contribuye con nuevos códigos, condiciones y experiencias a los procesos de elaboración identitaria de las juventudes. La globalización ha roto la homogeneidad de las culturas y por consiguiente, la inmovilidad de los roles. En este contexto, las identidades han tomado más relevancia, lo que incluso se aprecia en las políticas que deben considerar la diversidad de actores y poblaciones, así como en el énfasis surgido en relación a las identidades nacionales.

El período juvenil y la representación del tiempo de ser joven tienen valores distintos en las sociedades, estratos socioeconómicos y culturas, sus formas de productividad, participación y expresión. En la actualidad, las dos generaciones están preparándose permanentemente y deben considerar la flexibilidad frente a los cambios por sobre la irreversibilidad de los pasos dados. La incertidumbre es un elemento que se incorpora al desarrollo, pues ya no puede considerarse que la juventud vaya hacia un horizonte seguro y predefinido traducido en un proyecto de futuro. La articulación de las fuentes de la identidad se sustenta cada vez menos en la linealidad del desarrollo asentada en el patriarcado. Este conlleva la inequidad de género y la discriminación por edad como insumos organizadores de la identidad y las representaciones sociales de los sujetos juveniles. Toman importancia los peldaños que se insertan en un sentido y esperanza de vida, para lo que es necesario hacer frente a la redistribución del poder que implica la participación juvenil auténtica, la equidad de género y abrir los canales para la colaboración intergeneracional que valoriza a la juventud ciu- 
dadana, su flexibilidad para incorporar los conocimientos y aportar a la innovación.

Los procesos de individuación, las identidades flexibles, multicéntricas, la individualización, el peso de la exclusión y la discriminación, son aspectos a considerar en las estrategias de abordaje de las juventudes. Desaparecen las transiciones clásicas y dejan de ser irreversibles. Tiempo y espacio juegan roles diversos. Las sociedades se encuentran inmersas en procesos acelerados que sitúan a los jóvenes en nuevas condiciones y determinan el desarrollo de diversas subjetividades y prácticas. Para enfrentar el desafío de construir sociedades inclusivas es necesario contar con legislación, políticas públicas e institucionalidad orientadas al pleno reconocimiento de las capacidades y derechos de las juventudes y las medidas necesarias para su cumplimiento. Se requiere hacer frente a la redistribución del poder que implica la participación juvenil auténtica y abrir los canales para la colaboración intergeneracional que valoriza a la juventud ciudadana, su flexibilidad para incorporar los conocimientos y aportar a la innovación.

SANTIAGO (CHILE), AGOSTO 2010

RECIBIDO: AGOSTO 2010

ACEPTADO: OCTUBRE 2010

\section{REFERENCIAS BIBLIOGRÁFICAS}

Bajoit, GuY (2004): «Exposición analítica de los resultados de la cuarta encuesta nacional de juventud». Santiago.

Bajoit, GuY (2003): Todo cambia. Análisis sociológico del cambio social y cultural en las sociedades contemporáneas. Santiago: LOM Ediciones.

BECK, UlRICH (1998): La sociedad de riesgo. Hacia una nueva modernidad. Buenos Aires: Paidós.

BECK; BECK-GERNSHEIM (1995): El normal caos del amor. Las nuevas formas de la relación amorosa. Barcelona: Paidós.

De la Garza, Fidel; Iván Mendiola y Salvador Rábago (1977): Adolescencia marginal e inhalantes. México: Editorial Trillas.

GÓMEZ, MARCO TUlio (2003): «Comunidad e identidad juvenil: la identidad juvenil como construcción comunitaria». MARC ZIMMERMAN y GABRIELA BAEZA VENTURA (coords.): Estudios culturales centroamericanos en el nuevo milenio. Antigua: CIRMA, Rockefeller Foundation y Fundación Friedrich Ebert. 
KRAUSKOPF, DiNA (2007): «Dimensiones y pertenencias en la construcción de las identidades juveniles en Centroamérica». MARC ZiMMERMAN y GABRIELA BAEZA VENTURA (coords.): Estudios culturales centroamericanos en el nuevo milenio. Antigua: CIRMA, Rockefeller Foundation y Fundación Friedrich Ebert.

(2003): «Proyectos, incertidumbre y futuro en el período juvenil». Archivos Argentinos de Pediatría №101 (6). Buenos Aires.

- (1996): «Reflexiones y precisiones sobre la identidad y la importancia de la perspectiva sociocultural en los estudios de población». En Cultura y población en América Latina. San José: FLACSO y EAT-FNUAP. (1984): Adolescencia y educación. San José: EUNED.

LANDA, HOMERO y TANIA CRUZ (2006): «Juventudes en la posmodernidad mexicana». Revista de Estudios sobre Juventud, Año 10, N²4. México: IMJ.

LECHNER, NorberT (1998): «Condiciones de gobernabilidad democrática en América Latina». En Chile 97. Análisis y opiniones. Santiago: FLACSO.

LIEBEL, MANFRED (1992): Mala onda. La juventud popular en América Latina. Managua: Ediciones Nicarao.

LUNGO, IRENE (2003): «Construcción de los imaginarios sociales en jóvenes urbanos miembros de organizaciones de izquierda». MARC ZIMMERMAN y GABRIELA BAEZA VENTURA (coords.): Estudios culturales centroamericanos en el nuevo milenio. Antigua: CIRMA, Rockefeller Foundation y Fundación Friedrich Ebert.

MAfFesoli, Michel (2001): El instante eterno. El retorno de lo trágico en las sociedades posmodernas. Buenos Aires: Paidós.

MARTÍN-BARBERO, JESÚS (1998): «Jóvenes: des-orden cultural y palimpsestos». En Humberto Cubides, María Cristina LAVERde y Carlos EDUARDO VALDERRAMA: Viviendo a toda. Jóvenes, territorios culturales y nuevas sensibilidades. Bogotá: Siglo del Hombre Editores.

POITEVIN, RENÉ (2003): «La juventud, la educación y el trabajo en Guatemala». MARC ZimMERMAN y GABRIELA BAEZA VENTURA (coords.): Estudios culturales centroamericanos en el nuevo milenio. Antigua: CIRMA, Rockefeller Foundation y Fundación Frederich Ebert.

Reguillo, Rossana (1999): «La ética de lo efímero o los compromisos itinerantes». En: Apuntes. Lima: Casa de la Juventud.

SAlAZAR, AlONSO (1998): «Juventud y violencia». En JAIME ARTURO PADILlA (compilador): La constitución de lo juvenil. México: Causa Joven.

SERNA, LESLIE (1998): «Globalización y participación juvenil». Revista de Estudios sobre Juventud, cuarta época, Año 5. México: IMJ.

Xón, MARÍA JACINTA (2003): «El conflicto de la identidad cultural y etnia entre jóvenes indígenas y no indígenas en Guatemala». MARC ZIMMERMAN y GABRIELA BAEZA VENTURA (coords.): Estudios culturales centroamericanos en el nuevo milenio. Antigua: CIRMA, Rockefeller Foundation y Fundación Friedrich Ebert. 